Research Centre for Ageing,

Welfare and Labour Market Analysis

University of St.Gallen

\title{
Zu einigen Problemen der direkten Volksrechte
}

Gebhard Kirchgässner

SCALA Policy Paper No. 1/2009 
St. Gallen Research Centre for Ageing, Welfare and Labour Market Analysis (SCALA) University of St. Gallen (HSG)

Varnbüelstrasse 19

$\mathrm{CH}-9000$ St. Gallen

tel.

$+41(0) 712243138$

fax

$+41(0) 712243135$

scala@unisg.ch

http://www.scala.unisg.ch 


\title{
Zu einigen Problemen der direkten Volksrechte
}

\author{
von \\ GEBHARD KIRCHGÄSSNER \\ Universität St. Gallen \\ Schweizerisches Institut für Aussenwirtschaft und \\ Angewandte Wirtschaftsforschung (SIAW-HSG), \\ Leopoldina and CESifo
}

\begin{abstract}
In this paper several problems of direct popular decisions are discussed. In the first part, we consider problems related to the functioning of direct democracy. It makes only sense as a political system if it is a continuous process and if not only sometimes single questions are brought to a referendum. Then, the relation between direct democracy and the unanimous rule is discussed, a subject of special relevance ro the European Union, before we consider the role of quorums. In the second part, some areas are considered where conflicts might arise. The results of initiatives might be incompatible with individual human rights or might endanger fiscal sustainability, and referenda might impede economic reforms. All these problems do, however, not justify a general rejection of direct popular rights. Thus, we conclude by listing several points that should be observed in order to safeguard the well functioning of direct democracy.
\end{abstract}

Vortrag im Rahmen der Wissenschaftstagung des Deutschen Instituts für Sachunmittelbare Demokratie, Dresden, 25. Oktober 2008 - Überarbeitete schriftliche Fassung, Februar 2009.

Keywords: $\quad$ Direct democracy, referendum, initiative, human rights, economic reforms, fiscal sustainability.

JEL Classification: H11

Anschrift:

Prof. Dr. Gebhard Kirchgässner

University of St. Gallen

SIAW-HSG, Bodanstrasse 8

CH-9000 St. Gallen

Switzerland

Gebhard.Kirchgaessner@unisg.ch 


\section{Einleitung}

[1] Die Auswirkungen direkter Volksrechte sowie die Diskussionen um sie machen teilweise einen recht merkwürdigen Eindruck. So ist die Europäische Verfassung im Jahr 2005 an Volksabstimmungen in zwei Ländern, Frankreich und den Niederlanden, gescheitert. Ihre modifizierte Version, der Vertrag von Lissabon, droht, falls nicht eine zweite Abstimmung das Ergebnis vom 18. Juni 2008 noch umdreht, am Referendum in Irland zu scheitern. Es scheint, als ob die direkte Demokratie der Einigung Europas im Wege stünde. Dennoch wird sie aus unterschiedlichsten Gründen von den verschiedensten Seiten gefordert.

[2] Einer derjenigen, die Referenden über grundlegende Fragen der Europäischen Union gefordert haben, ist der frühere bayerische Ministerpräsident EDMUND STOIBER. ${ }^{1)}$ Dabei hat er sich nicht nur gegen die Ausweitung direkter Volksrechte in ,seinem‘ Bayern gesträubt, sondern auch mitgeholfen, dass sie wieder eingeschränkt wurden. ${ }^{2)}$ Ganz offensichtlich ging es ihm hier um den opportunistischen Einsatz direkter Volksrechte zur Erreichung eines bestimmten Ziels und nicht um ein generelles Bemühen um mehr direkte Mitsprache der Bürgerinnen und Bürger. Schließlich war auch er ordentliches Mitglied jener Gemeinsamen Verfassungskommission, die nach der Wiedervereinigung Deutschlands Anpassungen des Grundgesetzes diskutierte und in der insbesondere die CDU/CSU verhinderte, dass direkte Volksrechte auf Bundesebene eingeführt wurden. ${ }^{3)}$

[3] Aber auch Wissenschaftler sind gegen solche Versuchungen nicht gefeit. So fordert z.B. R. VAubeL (2000, 2001) Referenden über bestimmte Angelegenheiten der Europäischen Union, vermutlich weil er glaubt, dass diese Entscheidungen dann eher seinen Vorstellungen entsprechen würden. Gleichzeitig tritt er vehement für die weitgehende Beibehaltung des Einstimmigkeitsprinzips ein (2005). Wie unten gezeigt wird, schließt sich beides jedoch weitgehend aus, falls der Status Quo der europäischen Union nicht zementiert werden soll. Auch wäre R. VAUBEL wohl kaum dafür, direkte Volksrechte in der Europäischen Union generell zuzulassen, da dann die von ihm (2000) begrüßten Entscheide der Europäischen Union und des Europäischen Gerichtshof zur Stärkung des Wettbewerbs ebenfalls Volksabstimmungen unterworfen werden könnten. Wie das Beispiel der Schweiz zeigt, ist bei solchen Abstimmungen mit Ergebnissen zu rechnen, die den Vorstellungen einer weitgehenden Privatisierung und Deregulierung traditionell staatlicher Aktivitäten diametral zuwiderlaufen. Die Stimmbürgerinnen und Stimmbürger haben in der Schweiz nicht zufällig Vorlagen in dieser

1. Siehe z.B.: Stoiber wirbt für Volksabstimmung über die europäische Verfassung, Welt Online vom 31. Juli 2005. (http://www.welt.de/print-welt/article331451/Stoiber_wirbt_fuer_Volksabstimmung_ueber_die_ europaeische_Verfassung.html (10/12/08)); ein anderes Beispiel ist die von der SPÖ im Wahlkampf des Jahres 2008 erhobene Forderung, über EU-Verträge in Zukunft das Volk entscheiden zu lassen.

2. Seine Regierung hat im Jahr 2000 dafür gesorgt, dass eine mehr bürgerfreundliche Regelung der äußerst restriktiven Bestimmungen für Volksbegehren im Freistaat Bayern durch den (im Wesentlichen von der CSU bestimmten) Bayerischen Verfassungsgerichtshof als verfassungswidrig abgelehnt wurde. Auch schon früher hat die CSU veranlasst, dass die direkten Volksrechte erschwert werden, indem im Jahr 1968 die Eintragungsfrist für Volksbegehren von vier auf zwei Wochen reduziert wurde.

3. Siehe hierzu T. ScHмACK-RESCHKE (1997) sowie Bundestagsdrucksache 12/6000. 
Richtung mehrfach abgelehnt, selbst wenn neben den bürgerlichen Parteien auch die Sozialdemokraten dafür eintraten. Die Bevölkerung (vermutlich nicht nur) der Schweiz wünscht sich deutlich mehr Staat, als der von vielen Ökonomen vertretenen wirtschaftsliberalen Konzeption entspricht. Nicht umsonst ist daher die Schweiz eher ein Nachzügler in bezug auf Privatisierung und Deregulierung solcher Aktivitäten, und dafür wird, gerade auch von Ökonomen, hauptsächlich die direkte Demokratie verantwortlich gemacht. ${ }^{4)}$

[4] Aber auch in der Schweiz sind weder die Funktionsweise der direkten Volksrechte noch die Diskussion darüber völlig unproblematisch. So standen gerade in jüngerer Zeit Initiativen zur Abstimmung, die mit individuellen Menschenrechten in Konflikt stehen. Am 8. Februar 2004 wurde eine Initiative „Lebenslange Verwahrung für nicht therapierbare, extrem gefährliche Sexual- und Gewaltstraftäter“ angenommen, die im Gegensatz zur Europäischen Menschenrechtskonvention (ERMK) steht, da sie die Überprüfungsmöglichkeiten für die Verwahrung solcher Täter weitgehend ausschließt. Und obwohl der damalige Justizminister CHRIsTOPH BLOCHER hinter dem Anliegen dieser Initiative stand, war es auch ihm nicht möglich, dem Parlament ein Gesetz vorzuschlagen, welches eine ERMK-konforme Umsetzung ermöglichen würde. ${ }^{5)}$ Und am 1. Juni 2008 wurde eine Volksinitiative „Für demokratische Einbürgerungen“" abgelehnt, die nach dem Willen ihrer Verfechter Urnenabstimmungen über Einbürgerungen wieder möglich machen sollte, nachdem das Bundesgericht diese am 9. Juli 2003 in zwei Entscheidungen (BGE 129 I 217 sowie BGE 129 I 232) als verfassungswidrig erklärt hatte, da sie dem Anspruch auf rechtliches Gehör sowie auf Begründung der Ablehnung eines Gesuchs nicht genügten und damit im Widerspruch zum Willkürverbot des Artikels 9 der Bundeverfassung stünden.

[5] Hinter dieser Initiative stand die rechtsbürgerliche Schweizerische Volkspartei (SVP), die in der Vergangenheit mehrfach Wahl- und Abstimmungskampagnen mit ausländerfeindlichen Parolen bestritten hat, was im Jahr 2007 sehr negative Reaktionen im Ausland hervorgerufen hat. ${ }^{6)}$ Dabei vertritt diese Partei und insbesondere ihr langjähriger faktischer Führer, Altbundesrat Christoph BlOCHER, die Auffassung, dass die Ergebnisse von Volksabstimmungen höher zu gewichten sind als das Völkerrecht, denn: "Es darf nicht sein, dass ,höheres Recht" oder ,internationales Recht“ oder ,Völkerrecht‘ das demokratisch bestimmte Recht der eigenen Staatsbürger leichtfertig beschränkt oder gar außer Kraft setzt."7)

[6] Diese Beispiele zeigen, dass wir einerseits erhebliche Probleme mit Volksentscheiden haben, dass diese Probleme aber andererseits zum Teil gar nicht gesehen werden bzw. dass ex-

4. Siehe hierzu z.B. S. BORNER (2005).

5. Siehe hierzu: Verwahrungsinitiative weiter abgeschwächt: Doch Dilemma zwischen EMRK und Volkswillen bleibt, Neue Zürcher Zeitung Nr. 275 vom 24. November 2005, S. 13.

6. Siehe hierzu z.B.: Schweiz als schwarzes Schaf: Wie nie zuvor berichten ausländische Medien über den Wahlkampf - fast nur negativ, NZZ am Sonntag vom 14. Oktober 2007, S. 13, sowie: Endlich, die Schweiz holt auf, DIE ZEIT Nr. 43 vom 18. Oktober 2007, S. 11.

7. C. BlOCHER, 1. August-Rede 2007 in Schwarzenburg, http://www.blocher.ch/de/index.php?item=reden\&id=28 (12/01/09)). 
trem naiv (und/oder ideologisch) argumentiert wird. In dieser Arbeit sollten daher einige dieser Probleme aufgezeigt und diskutiert werden. Dabei geht es zum einen um die Funktionsweise der direkten Demokratie (Abschnitt 2), zum anderen um tatsächliche oder vermeintliche Konfliktfelder, die sich durch die direkten Volksrechte ergeben (Abschnitt 3). Dabei werden die einzelnen Abschnitte bzw. Unterabschnitte durch Thesen eingeleitet, die gleichzeitig eine Zusammenfassung bieten. Die Arbeit schließt mit einer Auflistung jener wichtigen Punkte, die sich aus unserer Diskussion ergeben und zu beachten sind, damit ein direkt-demokratisches System sinnvoll funktionieren kann (Abschnitt 4).

\section{Zur Funktionsweise der direkten Demokratie}

[7] In diesem Abschnitt geht es zunächst einmal darum deutlich zu machen, dass eine direkte Demokratie nur dann ein sinnvolles politisches System darstellt, wenn sie als dauerhafter Prozess ausgestaltet ist und man nicht nur gelegentlich einzelne Fragen ,herauspickt ‘ und den Bürgerinnen und Bürgern zur Abstimmung vorlegt (Abschnitt 2.1). Danach geht es um die vor allem für den Bereich der Europäischen Union bedeutsame Frage, wie sich die direkte Demokratie zur Einstimmigkeitsregel verhält (Abschnitt 2.2), bevor in Abschnitt 2.3 die Rolle von Quoren diskutiert wird. Dabei wird unter ,direkter Demokratie‘ immer ein ,halb-direktes System' verstanden, in welchem die repräsentative Demokratie durch direkte Volksrechte ergänzt wird. Ein rein direktes System, wie es heute noch in vielen schweizerischen Gemeinden und in den beiden Landsgemeindekantonen Appenzell-Innerrhoden und Glarus besteht, steht hier nicht zur Diskussion und ist auch für die Probleme, die sich z.B. in Zusammenhang mit der Europäischen Union ergeben haben, irrelevant.

[8] Bevor wir uns jedoch mit den Problemen der direkten Volksrechte näher befassen, soll zunächst klar gestellt werden, was hier behandelt werden soll. Zum einen geht es ausschließlich um Entscheidungen über Sachfragen; direkte Wahlen von Regierungen werden hier nicht betrachtet. Zweitens gilt es, vier verschiedene Arten von Volksabstimmungen zu Sachfragen zu unterscheiden:

(i) Kontrollierende Referenden: Vom Parlament verabschiedete Gesetze, Verfassungsbestimmmungen oder Ausgabenprojekte (,Finanzreferendum') müssen den Bürgern zur Abstimmung vorgelegt werden, bevor sie rechtskräftig werden können. Solche Referenden können obligatorisch oder fakultativ sein; im letzteren Fall müssen sie dann durchgeführt werden, wenn eine bestimmte Anzahl von Bürgern dies verlangt. Diese Referenden sollen ausschließen, dass die Regierenden Gesetze oder Ausgabenprojekte gegen den Willen der (Mehrheit der) Bürgerinnen und Bürger beschließen können. Sie helfen, ein Kartell der Regierenden gegen die Bürger zu verhindern.

(ii) Gesetzes- und Verfassungsinitiativen: Hier geht die Initiative vom Volk aus: Die Regierenden werden gezwungen, Gesetze zu beschließen und Maßnahmen zu ergreifen, die sie von sich aus nicht beschließen bzw. ergreifen würden. Solche Gesetze können z.B. dazu dienen, die Befugnisse von Regierung und Verwaltung oder Privilegien, welche sich die Parlamentarier selbst verschaffen, einzuschränken. 
(iii) Plebiszitäre Referenden (Plebiszit): Hier beschließen die Regierung und/oder das Parlament von sich aus, bestimmte Gesetzesvorlagen dem Volk zu unterbreiten, um für diese eine besondere Legitimation zu erhalten.

(iv) Konsultative Referenden: Bei diesen befragen die Regierenden die Bevölkerung in einer wichtigen Angelegenheit um ihre Meinung.

In Deutschland dreht sich die Diskussion vor allem um Initiativen, wobei das Einreichen einer Initiative als 'Volksbegehren' und die Entscheidung darüber als 'Volksentscheid' bezeichnet wird. Neben ihnen sind aber Referenden mindestens genauso wichtig, um den Bürgerinnen und Bürgern die Durchsetzung ihrer Interessen zu ermöglichen; beide stellen wirksame Mittel dar, die Regierung zu Rechtsnormen zu veranlassen, die ihren Wünschen entsprechen. Wir werden uns im Folgenden daher im Wesentlichen mit diesen beiden Arten von Volksabstimmungen beschäftigen.

\subsection{Direkte Demokratie als permanenter Prozess}

These 1: Es macht kaum Sinn, eklektisch einzelne Volksabstimmungen anzusetzen bzw. ad hoc einzelne Entscheidungen den Stimmbürgern zu überlassen. Direkte Demokratie wird in aller Regel nur dann zu befriedigenden Ergebnissen führen, wenn Abstimmungen regelmäßig durchgeführt werden und wenn die Frage, ob eine Abstimmung durchgeführt wird, nicht im Ermessen der Regierenden liegt, sondern entweder durch die Verfassung vorgeschrieben ist (obligatorisch) oder im Ermessen der Stimmbürger liegt (fakultativ).

[9] Wie die obigen Beispiele zeigen, fordern Politiker (aber nicht nur sie) in (fast) rein repräsentativen Systemen in bestimmten Fällen gerne Volksabstimmungen, wenn sie erwarten, ihre Ziele damit besser durchsetzen zu können. Dabei geht es um ad hoc angesetzte Plebiszite. Mit einem generellen Bekenntnis zur Einführung direkter Volksrechte hat dies im Allgemeinen wenig zu tun. Die Motivation dazu kann sein, dass man eine negative Entscheidung wünscht, die Verantwortung dafür aber nicht übernehmen will, oder dass man sich durch die Abstimmung für das eigene Handeln eine zusätzliche Legitimation erhofft. Wie verschiedene Beispiele in Frankreich zeigen, kann letzteres jedoch schief gehen. So trat Präsident Charles de GAUlLE am 28. April 1969 zurück, nachdem er am Tag zuvor eine von ihm selbst angesetzte Abstimmung über ein Gesetz zur Schaffung von Regionen und zur Erneuerung des Senats verloren hatte. Und auch Präsident JAQUES CHIRAC scheiterte mit seinem Versuch, sich durch eine Abstimmung über die Europäische Verfassung zusätzliche Legitimation zu verschaffen. In beiden Fällen ging es den Stimmbürgern weniger um das zur Abstimmung stehende Problem, sondern darum, ihrem Unmut über die Politik des französischen Präsidenten auf diese Art und Weise Luft zu verschaffen. ${ }^{8)}$ Mit solchen Reaktionen ist immer

8. Siehe z.B. S. TOMIK, der ,politische Unfall‘, FAZ.NET (http://www.faz.net/s/Rub99C3EECA60D84C08AD6B3E60C4EA807F/Doc E48B2A50994C2461482036 C69CB5BEDE1 ATpl Ecommon Scontent.html (12/01/09)). 
dann zu rechnen, wenn es sich um Plebiszite handelt, die überdies selten durchgeführt werden. Die direkten Volksrechte werden damit ihrer eigentlichen Funktion entleert und in gewisser Weise missbraucht, und zwar sowohl von den Regierenden, welche die Plebiszite ansetzen, als auch von den Stimmbürgern, die diese weitgehend unabhängig von der zu entscheidenden Frage als Mittel zum Protest einsetzen. Bei regelmäßig durchgeführten Referenden sowie bei Abstimmungen über Initiativen kann ein solches Verhalten zwar nicht völlig ausgeschlossen werden, aber es ist sehr viel weniger wahrscheinlich.

[10] Aber nicht nur dann, wenn es sich um einzeln angesetzte Plebiszite handelt, sondern auch bei 'normalen' Referenden können die Resultate im Widerspruch zu dem sein, was man sich wünscht bzw. für wirtschaftlich sinnvoll hält. In jüngerer Zeit gilt dies vor allem für Anliegen der Deregulierung und der Privatisierung von Aktivitäten, die traditionell als Staatsaufgaben galten. Seit den achtziger Jahren hat sich hier unter Führung von Ökonomen ein erheblicher Wandel vollzogen, wobei in Westeuropa die Europäische Union die treibende Kraft war. Dabei zeigte sich freilich, dass Privatisierungen ein sehr viel schwierigeres Unterfangen sind, als man sich dies in den siebziger und achtziger Jahren vorstellte. Zudem ist nach der Privatisierung nicht alles erledigt. Vielmehr bedarf es in vielen Fällen aufwendiger Regulierungsmechanismen, um letztlich ein befriedigendes Ergebnis zu erzielen. ${ }^{9)}$ Das mangelnde Vertrauen darauf, dass dies erreicht werden kann, hat in jüngerer Zeit in der Schweiz mehrere von der Politik geplante Privatisierungen scheitern lassen. So wurde z.B. im Kanton BaselStadt am 19. November 1995 die Privatisierung der Müllverbrennungsanlage in einer Volksabstimmung verworfen, obwohl im Parlament hierfür eine deutliche Mehrheit vorhanden war. ${ }^{10)}$ Eine weitere ,Privatisierungsverweigerung fand am 10. Juni 2001 in Zürich statt, als die Stimmbürger mit 51.4 Prozent die Umwandlung der Elektrizitätswerke des Kantons Zürich in eine Aktiengesellschaft und damit deren Privatisierung ablehnten. ${ }^{11)}$ Offensichtlich möchte die (politisch aktive) Bevölkerung bestimmte Angelegenheiten nicht gerne aus der Hand geben, bzw. sie vertraut nicht ohne weiteres darauf, dass bei einer Privatisierung die Qualität der angebotenen Leistungen, mit der sie nach Umfrageergebnissen heute zufrieden ist, erhalten bleibt. ${ }^{12)}$ Schließlich kann es, wie z.B. D. Bös (1989) herausgearbeitet hat, bei einer Privatisierung durchaus um ein Spannungsverhältnis zwischen Effizienz und Qualität gehen, und die wesentlichen Argumente zugunsten einer Privatisierung sind in diesen Fällen eher politischer (bzw. ideologischer) als ökonomischer Natur. ${ }^{13)}$

9. Siehe hierzu auch W.W. POMMEREHNE (1990) sowie F. SCHNEIDER (1998).

10. Siehe: Basel gegen Privatisierung des Kehrichtwesens, Neue Zürcher Zeitung Nr. 270 vom 20. November 1995, S. 13.

11. Siehe: Elektrizitäts-Neuordnung knapp verworfen: Gehaltene Ernüchterung bei Regierung, EKZ und Axpo, Neue Zürcher Zeitung Nr. 133 vom 11. Juni 2001, S. 41.

12. Siehe: Zufriedene Stromkunden sagen Nein: Analyse Abstimmungsergebnisse zur EKZ-Vorlage, Neue Zürcher Zeitung Nr. 198 vom 28. August 2001, S. 41.

13. Siehe hierzu auch M. PRISCHING (1988), der darauf hinweist, dass es sich bei der Privatisierung häufig (auch) um symbolische Politik handelt. Dies muss nicht gegen diese sprechen, beleuchtet jedoch einen Aspekt, der in ökonomischen Diskussionen meist übersehen wird. 
[11] Aber nicht nur Privatisierungen sind gescheitert, mit der Ablehnung des Elektrizitätsmarktgesetzes vom 22. September 2002 ist in der Schweiz auch ein wichtiges Deregulierungsvorhaben von den Stimmbürgerinnen und Stimmbürgern zurückgewiesen worden. ${ }^{14)}$ Dabei war hier - genau wie bei den gescheiterten Privatisierungen - neben der Mehrheit der politischen Repräsentanten auch die Mehrheit der Ökonomen für diese Änderung. Es ist - zumindest prima facie - ein merkwürdiges Ergebnis, dass die Stimmbürgerinnen und Stimmbürger den Bereich jener Aufgaben, welche dem Staat zugewiesen werden, weiter ausgedehnt sehen möchten, als es den Vorstellungen der (Mehrheit der) sie repräsentierenden Politiker entspricht. Dies ist zumindest für den politischen Ökonomen erstaunlich, weil eine der Annahmen in der ökonomischen Theorie der Politik bzw. der Bürokratie ist, dass die Politiker und die Bürokraten ein Interesse daran haben, die Staatstätigkeit stärker auszudehnen, als dies den Interessen ihrer Wählerinnen und Wähler entspricht.

[12] Offenbar vertrauen die Bürger darauf, dass ihr politischer Einfluss auf staatliche Unternehmen in dieser Hinsicht stärker zu ihrem Vorteil gereicht, als dies bei privaten Aktiengesellschaften der Fall wäre. Als Staatsbürger hat man in einer direkten Demokratie diese Entscheidungen der Stimmbürger zu respektieren. Soweit wir als Ökonomen davon überzeugt sind, dass eine (rein) privatwirtschaftliche Lösung in solchen Fällen effizienter gewesen wäre, und soweit wir deshalb auch dafür eingetreten sind, stellt sich für uns freilich die Frage, weshalb die Stimmbürger, denen wir in unserer Theorie üblicherweise rationale Erwartungen unterstellen, in diesen Fällen offensichtlich eine andere Auffassung vertreten als wir und die von uns beratenen Politiker. Wie G.J. STIGLER (1979) hervorgehoben hat, sollte man vorsichtig damit sein, dies (ad hoc) auf mangelnde und/oder verzerrte Information der Stimmbürgerinnen und Stimmbürger über die ,wahren' Nutzen und Kosten solcher politischer Maßnahmen zu schieben.

[13] Insofern liegen A. BRunETTi (1997) und S. BORNER (2005) mit ihrer Kritik an der direkten Demokratie nicht falsch, wenn man daraus auch nicht ihre Schlüsse ziehen muss. Auf jeden Fall aber sollte man die direkte Demokratie nicht nach dem Ausgang einzelner Entscheidungen beurteilen, sondern nach der zu erwartenden durchschnittlichen Qualität der Gesamtheit aller Entscheidungen. Und als Vergleich sind die tatsächlichen Situationen in repräsentativen Demokratien heranzuziehen, nicht irgendwelche Idealbilder. Vergleiche tatsächlicher Situationen mit Idealbildern haben keine Aussagefähigkeit. Auch dann aber spricht, wie z.B. in G. KIRCHGÄSSNER, L.P. FELD und M.R. SAVIOZ (1999) herausgearbeitet wurde, noch viel für die direkte Demokratie.

14. Dabei ist interessant, dass die rechtsbürgerlichen Wähler der SVP die Vorlage noch stärker verwarfen als die SP-Sympathisanten. Insofern lässt sich dieses Ergebnis nicht auf ein einfaches Rechts-Links-Schema ,weniger، vs. ,mehr Staat‘ zurückführen. Siehe hierzu: SP stimmte wirtschaftsliberaler als SVP: Vox-Analyse zu den Abstimmungen vom 22. September, Neue Zürcher Zeitung Nr. 265 vom 14. November 2002, S. 13. - Bei dieser Ablehnung dürfte die Entwicklung in Kalifornien eine wesentliche Rolle gespielt haben, auch wenn die Bedingungen nicht vergleichbar sind. Zur Situation in Kalifornien siehe z.B. R.T. CROw (2002). 


\subsection{Direkte Demokratie und Einstimmigkeitsregel}

These 2: In einem föderalen System führt die Einstimmigkeitsregel bei Entscheidungen, die durch Volksabstimmungen getroffen werden, fast notwendigerweise zum Stillstand. Dies gilt auch für die Europäische Union. Sollen Entscheidungen über deren künftige Verfassung durch Volksentscheide getroffen werden, dann sollten solche Abstimmungen in allen Mitgliedsländern durchgeführt werden, und es sollte neben einem qualifizierten Mehr der Gesamtbevölkerung auch ein qualifiziertes Mehr der beteiligten Staaten genügen, um die Verfassung in Kraft zu setzen.

[14] In der Europäischen Union gilt in weiten Bereichen und insbesondere dann, wenn es um Verfassungsfragen geht, die Einstimmigkeitsregel. Der große Vorteil dieser Regel ist, dass sie dem Pareto-Kriterium genügt: Keiner kann durch eine Entscheidung der anderen gegen seinen Willen schlechter gestellt werden. Sie räumt damit jedem Entscheidungsträger eine Vetoposition ein.

[15] Mit zunehmender Zahl der Beteiligten wird die Einstimmigkeitsregel jedoch immer problematischer. Das Problem ist nicht nur, dass dann die ,normalen` Einigungskosten ansteigen, sondern vor allem, dass damit die Gefahr strategischen Verhaltens zunimmt: Ist sich jemand bewusst, dass er/sie für eine positive Entscheidung unbedingt benötigt wird, ergibt sich die Möglichkeit, diejenigen, die durch Annahme eines Vorschlags besonders begünstigt werden, ,auszubeuten'. Da sich bei Einstimmigkeit jedermann in dieser strategisch günstigen Situation befindet, steigen die Einigungskosten bei Anwendung dieser Regel im laufenden politischen Prozess stark an. Dies gilt umso stärker, je größer der Kreis der an einer Abstimmung Beteiligten ist. Es entsteht dann eine Situation, in der Entscheidungen immer schwieriger werden: Es kommt zur Blockadepolitik bzw. zum Reformstau. ${ }^{15)}$

[16] Auf dieses Problem hat bereits K. WicKsell (1896, S. 117) hingewiesen. Obwohl er von seiner theoretischen Konzeption her für das Einstimmigkeitsprinzip argumentierte, sah er die Probleme bei seiner praktischen Anwendung und hat deshalb für die entsprechenden Abstimmungen eine „qualifizierte Majorität, etwa von drei Vierteln, fünf Sechsteln oder gar neun Zehnteln der abgegebenen Stimmen“ vorgeschlagen.

[17] Bei einer überschaubaren Zahl Beteiligter kann man versuchen, dieses Problem durch Stimmentausch zu lösen, indem man sich dahingehend abspricht, dass man Projekte anderer auch dann unterstützt, wenn sie für einen selbst nicht vorteilhaft sind, vorausgesetzt, die anderen unterstützen im Gegenzug die für einen selbst wichtigen Projekte. Ein solcher Stimmen-

15. Es ergibt sich damit eine Situation, die gleichsam das Komplement zur Tragik der Allmende darstellt. Während dort zu viel Aktivität entfaltet wird, weil jeder ohne Zustimmung der anderen Betroffenen handeln kann, wird jetzt zu wenig (bzw. gar keine) Aktivität entfaltet, weil immer die Zustimmung Aller erforderlich ist. Siehe hierzu auch J.M. BUCHANAN und Y.J. YoON (2000) sowie aus politikwissenschaftlicher Sicht A. HÉRETIER (1999). 
tausch kann für alle von Vorteil sein. Er stellt auf jeden Fall die einzige Möglichkeit dar, in einer Gruppe mit divergierenden Interessen einstimmige Entscheidungen herbeizuführen. ${ }^{16)}$

[18] Stimmentausch setzt bindende Absprachen zwischen den Beteiligten voraus. Dies wird umso schwieriger, je grösser die Gruppe ist, auch weil die oben angesprochenen strategischen Motive dann immer stärker werden. Entscheiden die Bürgerinnen und Bürger, sind bindende Absprachen nicht möglich; sie sind frei, nach ihren eigenen Vorstellungen zu entscheiden. Dies wird immer wieder deutlich, wenn bei Wahlen Parteivorstände Absprachen zur gegenseitigen Unterstützung der Kandidaten treffen, die abstimmende Bevölkerung sich daran aber nicht hält. Daher ist expliziter Stimmentausch bei Abstimmungen nicht möglich; man kann allenfalls impliziten Stimmentausch versuchen, indem man bestimmte ,Pakete' schnürt. Wie die Erfahrung der Schweiz zeigt, ist dies jedoch sehr schwierig, und es scheitert häufig. Dies aber bedeutet, dass bei Volksabstimmungen die Einstimmigkeitsregel keinen Sinn macht; sie bewirkt vielmehr eine totale Blockade und damit eine Zementierung des Status quo.

[19] Wenn man überhaupt Veränderungen ermöglichen will, sind daher direkte Demokratie und Einstimmigkeitsprinzip nicht vereinbar; man muss sich entscheiden, was man will. Beharrt man auf beidem, muss man sich mit dem Vorwurf auseinandersetzen, dass man ganz bewusst eine Blockade herbeiführen will. Dies sollte man dann aber auch offen zugeben und die direkten Volksrechte nicht in dieser Weise missbrauchen.

\subsection{Die Rolle von Quoren}

These 3: Man kann versuchen, mit Quoren die Legitimation direkt-demokratischer Entscheide zu stärken, da Entscheide, die nur von wenigen Prozent der Bevölkerung getroffen werden, kaum auf allgemeine Akzeptanz stoßen dürften. Dabei ist jedoch folgendes zu berücksichtigen:

a) Wegen des damit verbundenen Anreizes zu strategischem Verhalten sind Beteiligungsquoren generell abzulehnen. Sie sind faktisch (sehr hohe) Zustimmungsquoren.

b) Wenn man Quoren (insbesondere für Verfassungsinitiativen) einführen will, sollten diese zumindest nicht höher sein, als die impliziten Zustimmungsquoren bei parlamentarischen Entscheidungen.

Die geforderten Unterschriftenzahlen zum Einreichen einer Initiative oder eines (fakultativen) Referendums sollten 5 bis maximal 10 Prozent der Stimmberechtigten nicht überschreiten.

[20] Um die Legitimität von Volksentscheiden zu erhöhen, wird häufig gefordert, dass eine Volksabstimmung nur dann gültig sein soll, wenn die Beteiligung ein bestimmtes Mindest-

16. Zum Stimmentausch siehe z.B. D.C. Mueller (2003, S. 104ff.). - Ein typisches Beispiel für solchen Stimmentausch sind Koalitionsverträge. 
quorum erreicht. In der Regel werden 50 Prozent gefordert. Entsprechende Regeln gibt es z.B. in Italien und in Hamburg, und dies fordert z.B. auch W. WitTMAnN (1998, S. 233) für die Schweiz. Damit soll verhindert werden, dass ,zufällige“ Mehrheiten eines kleinen, aber motivierten Teils der Bevölkerung Entscheidungen gegen den Willen des von der ganzen Bevölkerung gewählten Parlaments herbeiführen können. Entscheidungen des Volkes würden damit nur noch dann politisch offiziell zur Kenntnis genommen, wenn auch ein entsprechend großer Teil der Stimmberechtigten, auf jeden Fall über 25 Prozent, dahinter steht.

[21] Man würde zunächst vermuten, dass eine solche Regelung zu einer Erhöhung der Stimmbeteiligung führt, da die ,moralischen` Argumente, welche die Bürgerinnen und Bürger zur Stimmabgabe motivieren, dadurch verstärkt werden, wenn eine geringe Stimmbeteiligung die Gefahr nach sich zieht, dass eine vom Volk gewünschte Regelung politisch nicht umgesetzt wird. Tatsächlich aber ist das Gegenteil der Fall. Bei einem Beteiligungsquorum von 50 Prozent ist es für Gegner der Vorlage immer rational, sich nicht an der Abstimmung zu beteiligen, da dann im Extremfall dieses Beteiligungsquorum nur dann erreicht werden kann, wenn mehr als die Hälfte der Stimmberechtigten für die Vorlage eintritt. Dann aber würde diese in jedem Fall angenommen. Ansonsten aber kann man sie einfacher über eine mangelnde Stimmbeteiligung als über eine Mehrheit der Nein-Stimmen zu Fall bringen. Da eine Zustimmung von 50 Prozent aller Stimmberechtigten in den meisten Fällen sehr unwahrscheinlich ist, führt eine solche Regelung dazu, dass sich in (möglicherweise) zahlreichen Fällen zwar eine (deutliche) Mehrheit für eine Vorlage findet, die 50 Prozent-Schranke aber nicht erreicht wird. ${ }^{17)}$

[22] Die negativen Konsequenzen dieser Regelung haben sich in der Weimarer Republik gezeigt, ${ }^{18)}$ sie wurden aber auch in Italien deutlich, als am 18. April 1999 über eine Änderung des Wahlrechts abgestimmt wurde. Obwohl mit 91.7 Prozent die überwältigende Mehrheit der Abstimmenden für diese Änderung war, kam sie nicht zustande, da die Abstimmungsbeteiligung mit 49.6 Prozent knapp unter der vorgeschriebenen 50 Prozent-Marke lag.

[23] Darüber hinaus ist die hinter diesem Vorschlag stehende Argumentation selbst fragwürdig. Zum einen ist nicht unumstritten, dass eine hohe Wahl- bzw. Abstimmungsbeteiligung immer ein gutes Zeichen ist. Die Legitimität einer vom Volk getroffenen politischen Entscheidung ist durch eine niedrige Beteiligung nicht unbedingt in Frage gestellt. Zum anderen ist zu berücksichtigen, dass die Beteiligung auch bei Wahlen häufig sehr gering ist. Sie lag z.B. in der Schweiz selbst bei den Wahlen zum Nationalrat in den letzten beiden Jahrzehnten unter 50 Prozent. Damit aber ist völlig offen, worin die Legitimation bestehen sollte, dass z.B. die knappe Entscheidung eines mit nur wenig mehr als 40 Prozent der Stimmen gewählten Parlaments eine deutliche Entscheidung des Volkes, bei der jedoch die Beteiligungsgrenze von 50 Prozent knapp verfehlt wird, umstoßen kann.

17. Etwas abgeschwächt besteht der gleiche Anreiz zu strategischer Stimmenthaltung auch bei niedrigeren Beteiligungsquoren.

18. Siehe hierzu G. KirCHGÄSSNER, L.P. FELD und M.R. SAVIOZ (1999, S. 145ff.) 
[24] Will man dennoch ein Quorum einführen, dann sollte dies ein Zustimmungsquorum sein. Man könnte sich z.B. vorstellen, dass eine Entscheidung nur dann gültig ist, wenn mindestens 25 Prozent der Abstimmungsberechtigten zustimmen. ${ }^{19)}$ In diesem Fall hätten sowohl Anhänger als auch Gegner der Vorlage einen Anreiz sich an der Abstimmung zu beteiligen. Das Quorum sollte freilich nicht so hoch angesetzt werden, dass es faktisch eine höhere Beteiligung als bei den Wahlen erfordert. Für ein so hohes Quorum dürfte sich kaum eine Rechtfertigung finden lassen.

[25] Neben den Zustimmungs- bzw. Beteiligungsquoren sind jedoch auch die Quoren für die Unterschriftenzahlen relevant. In der Schweiz sind diese heute relativ niedrig: Man benötigt auf Bundesebene 100'000 Unterschriften für eine Initiative und 50'000 für ein Referendum. Dies sind etwas mehr als 2 bzw. 1 Prozent der Stimmberechtigten. Die Unterschriften müssen bei einem Referendum innerhalb von 6 Wochen, bei einer Initiative innerhalb von 18 Monate gesammelt werden. In den deutschen Bundesländern sind die Bestimmungen sehr viel restriktiver: In Bayern müssen z.B. zunächst 25'000 Stimmberechtigte einen Antrag stellen. Danach müssen innerhalb von 14 Tagen 10 Prozent der Stimmberechtigten das Volksbegehren unterschreiben, damit es (nach parlamentarischer Beratung) zur Abstimmung gelangen kann. ${ }^{20}$ )

[26] Derart restriktive Bestimmungen hebeln die Volksrechte weitgehend aus. Insofern ist es kein Wunder, dass es in Bayern nach 1945 ganze 17 Volksbegehren und nur 13 Volksentscheide gab. ${ }^{21)}$ In diesem Zusammenhang ist eine Untersuchung von J.G. MATSUSAKA (1995) interessant. Er zeigt für die amerikanischen Bundesstaaten, dass ein (statistisch) signifikanter Effekt des Initiativrechts nur dann gegeben ist, wenn weniger als 10 Prozent der Stimmberechtigten unterschreiben müssen. ${ }^{22)}$ Die heute z.B. in Bayern geltenden Bestimmungen sind genau wie die 50 Prozent-Beteiligungsregel in Hamburg Regelungen, welche die formal bestehenden Volksrechte derartig einschränken, dass sie fast wirkungslos werden. ${ }^{23)}$

\section{Potentielle Konfliktfelder der direkten Demokratie}

[27] Die Befürchtungen, die in Bezug auf die direkten Volksrechten geäußert werden, sind zwar zum Teil strategischer Natur, weil diese Rechte eine nicht erwünschte partielle Machtverschiebung von den gewählten Repräsentanten des parlamentarischen Systems zurück zum Souverän bewirken, sie sind zum Teil aber auch durchaus ernst zu nehmen. Dies gilt insbesondere für mögliche Konflikte mit dem Völkerrecht, wie sie in der Schweiz tatsächlich auftreten (Abschnitt 3.1). Aber auch für den Finanzvorbehalt kann man Argumente finden ( $A b$ schnitt 3.2). In beiden Fällen gilt jedoch, dass sich die Befürchtungen in erster Linie auf die

19. Dies gilt z.B. in Bayern für Verfassungsänderungen.

20. Siehe http://www.stmi.bayern.de/buerger/wahlen/volksbegehren/ (13/01/09).

21. Siehe ebenda.

22. Siehe hierzu auch E.R. GERBER (1999).

23. Das Gleiche gilt auch für Regelungen wie bisher in Hamburg, die erlauben, dass sich Parlament und Regierung über Volksentscheide hinwegsetzen. 
Wirkung von Initiativen beziehen, während Referenden davon überhaupt oder fast gar nicht betroffen sind. Gegen letztere wird andererseits eingewandt, dass sie eine Reformpolitik erschweren wenn nicht gar verunmöglichen (Abschnitt 3.3). Auf diese Befürchtungen soll im Folgenden eingegangen werden.

\subsection{Direkte Demokratie und Menschenrechte}

These 4: Ein grundlegendes und praktisch zwar zu bewältigendes, aber nicht wirklich lösbares Problem besteht in der Antagonie zwischen dem Rechtsstaats- und dem Demokratieprinzip. Beide können nicht gleichzeitig voll verwirklicht werden. Da die Kompetenzkompetenz beim Verfassungsgeber liegt und über die Verfassung mit Mehrheit (nicht einstimmig) entschieden wird, können demokratische Entscheidungen, welche ,unzulässig' in die individuellen Rechte eingreifen, nicht mit Sicherheit vermieden werden. Dies spricht für ein starkes Verfassungsgericht. Andererseits kann letzteres den legitimen Spielraum des demokratisch legitimierten Gesetzgebers selbst dort unverhältnismäßig stark einschränken, wo keine grundlegenden Menschenrechte tangiert sind. In der Verfassung muss daher eine Lösung gefunden werden, die eine Balance zwischen diesen beiden Prinzipien findet.

[28] Der moderne, in der abendländischen Tradition stehende Staat ist durch zwei Prinzipien gekennzeichnet, die in einem Spannungsfeld zueinander stehen: Demokratie und Rechtsstaatlichkeit. ${ }^{24)}$ So kann eine (extrem ausgebaute) direkte Demokratie dazu führen, dass vom Volk willkürliche Entscheide getroffen werden, die elementaren Menschen- bzw. Bürgerrechten, wie sie heute in der westlichen Welt weitgehend oder sogar allgemein akzeptiert sind und wie sie sich in der Deklaration der Menschenrechte der Vereinten Nationen finden, eindeutig widersprechen. Als Beispiel mögen die Einbürgerungsentscheidungen vom 4. Dezember 1997 in Pratteln und vom 12. März 2000 in Emmen dienen, bei denen Menschen türkischer bzw. jugoslawischer Herkunft trotz einer eindeutigen Empfehlung der Einbürgerungskommission an der Urne die Einbürgerung verweigert wurde. ${ }^{25)}$ Dies kann aber auch in einer rein repräsentativen Demokratie geschehen, wie die Beibehaltung (bzw. Reaktivierung) der Todesstrafe in den Vereinigten Staaten zeigt. Andererseits kann, wie das deutsche Beispiel zeigt, eine extrem weit ausgebaute Verfassungsgerichtsbarkeit dazu führen, dass das Parlament selbst mit überwiegender Mehrheit bestimmte Entscheidungen nicht mehr so treffen kann, wie sie in anderen (Rechts-)Staaten getroffen werden, auch wenn diese keineswegs den allgemein anerkannten

24. Siehe hierzu auch A. D’ATENA (1999). - Selbstverständlich bedarf die Demokratie eines bestimmten Regelwerks, damit überhaupt Entscheidungen zustande kommen und durchgesetzt werden können. Hierfür sind rechtliche Vorkehrungen notwendig. Dies ändert aber nichts am prinzipiellen Spannungsfeld zwischen beiden Prinzipien.

25. Siehe hierzu: Emmen sagt Nein zu 19 Einbürgerungsgesuchen, Neue Zürcher Zeitung Nr. 61 vom 13. März 2000, S. 10; Willkürliche Verweigerung der Einbürgerung: Baselbieter Verfassungsgericht hebt Pratteler Entscheide auf, Neue Zürcher Zeitung Nr. 76 vom 30. März 2000, S. 13; Unterschiedlich strenge Schweizermacher: Keine Beweise, aber Indizien der Diskriminierung, Neue Zürcher Zeitung Nr. 260 vom 7. November 2000, S. 13; A. Auer, Einbürgerung durch Volksentscheid? Verfassungsrechtliche Grenzen der direkten Demokratie, Neue Zürcher Zeitung Nr. 73 vom 27. März 2000, S. 13. 
Menschenrechten widersprechen. Dies zeigt sich z.B. daran, wie weitgehend das deutsche Bundesverfassungsgericht den Gesetzgeber selbst bei Fragen der Besteuerung bindet.

[29] Hinter diesen Beispielen stehen zwei unterschiedliche Verfassungstraditionen, eine ,liberale“ und eine ,demokratische‘, die sich nicht nur auf unterschiedliche ,Stammväter“ in der Zeit der Aufklärung berufen können, ${ }^{26)}$ sondern die auch an der gegensätzlichen Entwicklung in Deutschland (Preußen) und in der Schweiz im 19. Jahrhundert sehr gut illustriert werden können. Auch wenn viele mit der Französischen Revolution und ihrem Gleichheitsideal sympathisierten, hatte angesichts der Stärke der Fürstenhäuser das demokratische Prinzip in Deutschland zu Beginn des 19. Jahrhunderts keine Chance auf auch nur annähernde Verwirklichung. Gemäß dem monarchistischen Prinzip lag die entscheidende Gewalt bis zur Novemberrevolution am Ende des Ersten Weltkriegs im Jahr 1918 bei den Fürstenhäusern. ${ }^{27)}$ Dennoch hat sich in dieser Zeit, insbesondere im Gefolge der in Preußen durchgeführten ,SteinHardenbergschen Reformen', ein Verwaltungs- und Rechtsstaat entwickelt, welcher die Befugnisse der Monarchie insofern einschränkte, als auch das Handeln der Fürsten, insbesondere aber ihrer Verwaltungen, durch die in den ersten Jahrzehnten dieses Jahrhunderts erlassenen Verfassungen eingeschränkt wurde. ${ }^{28)}$ Dabei war es den Bürgern möglich, gegen die Überschreitung dieser Grenzen durch die Verwaltung vor Gericht zu klagen. Zwar wurde die Gerichtsbarkeit nach wie vor im Namen des Fürsten ausgeübt, ,aber die Rechtsprechung hatte sich dennoch ihre Unabhängigkeit von der Verwaltung erkämpft. Die Justiz wurde durch unabhängige, nur dem Gesetz unterworfene Beamte ausgeübt.“29)

[30] In der Schweiz dominierte dagegen mit der Ablösung der liberalen durch die radikalen Regierungen in den dreißiger und vierziger Jahren des 19. Jahrhunderts das demokratische gegenüber dem liberalen Prinzip: ${ }^{30)}$ Die ,freien` Schweizer waren gewillt, ihre politischen Angelegenheiten selbst zu regeln. Dies bedeutete nicht notwendigerweise die Einführung der direkten Demokratie: Um 1840 gab es zwar in sieben Kantonen eine Landsgemeinde und in sechs eine halbdirekte Demokratie, aber 11 Kantone hatten eine rein repräsentative Demokra-

26. F.A. V. HAYEK (1967, S. 11) nennt als Vordenker der liberalen Tradition u.a. David Hume, Adam Smith, Alexis de Toqueville, Immanuel Kant, Wilhelm von Humboldt und James Madison, als Vertreter der demokratischen Tradition Voltaire, Jean Jacques Rousseau sowie Marquis de Condorcet. Zur Charakterisierung der beiden Ansätze siehe z.B. F. SCHNABEL (1964, S. 128ff.).

27. Zum ,monarchistischen` Prinzip und seiner Anwendung in den verschiedenen Verfassungen in Deutschland siehe ebenfalls F. SCHNABEL (1964, S. 111ff.).

28. Eigentlich gehören zur Idee des Rechtsstaats, die Ende des 18. Jahrhunderts aufkam, drei Elemente, „die Hoffnung des Bürgertums auf eine von staatlicher Bevormundung freie Entfaltung des Marktes ..., das Bedürfnis nach Befreiung von absolutistischer Gängelung in Religions- und Bildungsfragen sowie der Anspruch auf politische Mitwirkung des dritten Standes, wenn auch dieses letztere Element in Deutschland ungleich schwächer ausgeprägt war als in Frankreich oder England.“ (M. STOLlEIS (1990, S. 367).) - Zu den einzelnen Reformen siehe T. NiPPERDEY (1983, S. 31ff.).

29. H. FEHR (1962, S. 275).

30. Siehe hierzu G. ANDrEy (1983, S. 267) sowie M. SCHAFFnER (1998). 
tie, während Neuenburg eine konstitutionelle Monarchie war. ${ }^{31)}$ Auch die neue Bundesverfassung des Jahres 1848 hatte nur wenige Elemente der direkten Demokratie; die wichtigsten heute auf eidgenössischer Ebene bestehenden Volksrechte, das fakultative Gesetzesreferendum und die Volksinitiative auf Teilrevision der Verfassung, wurden erst 1874 (im Rahmen der ersten Totalrevision) sowie 1891 in die Verfassung aufgenommen. Neben diesen demokratischen Rechten hatte der Rechtsstaat eine geringere Bedeutung: Insbesondere dort, wo eine Landsgemeinde existierte, d.h. wo jeder (männliche) Bürger seine Anliegen in den politischen Prozess einbringen konnte, machte es wenig Sinn, gegen politische Entscheidungen bei Gericht klagen zu wollen. ${ }^{32)}$ Diese Überzeugung ist bis heute unter der schweizerischen Bevölkerung weit verbreitet, und sie zeigt sich z.B. im Widerstand gegen die Einführung einer Verfassungsgerichtsbarkeit auf Bundesebene.

[31] Der Grundwiderspruch zwischen dem (häufig als ,Rule of Law`) bezeichneten Rechtsstaatsprinzip und dem Demokratieprinzip ist zwar pragmatisch regelbar, aber nicht wirklich lösbar. Wie immer die verwendete Argumentation zur Legitimation bestimmter Normen auch aussehen mag, es gibt letztlich nur positives Recht, d.h. es muss eine (menschliche) Instanz geben, die jegliches - und damit auch das grundlegende Verfassungsrecht - setzt. ${ }^{33)}$ Dies gilt insbesondere für den säkularen Staat. In modernen Gesellschaften geschieht dies mit Hilfe demokratischer Verfahren, wobei unterschiedliche Verfahren und Quoren auf unterschiedliche Bereiche angewendet werden können. So kann z.B. vorgesehen werden, dass bestimmte Elemente der Verfassung - im Sinne sog. ,Ewigkeitsklauseln` - nicht geändert werden dürfen, auch nicht mit jener Mehrheit, welche befugt ist, die Verfassung zu ändern. Damit können insbesondere die wesentlichen Menschen- und Bürgerrechte abgesichert werden. Das Verfassungsgericht hat dann die Aufgabe der Hüterin dieser Rechte und vertritt damit - auch gegenüber der demokratisch legitimierten gesetzgebenden Körperschaft - das Rechtsstaatsprinzip. Dies kann aber nicht verhindern, dass - ebenfalls auf demokratischem Weg - eine neue Verfassung in Kraft gesetzt wird, welche die demokratischen Mitwirkungsrechte und/oder die bürgerlichen Freiheitsrechte beschneidet, wie dies z.B. vor einigen Jahren in Liechtenstein (sogar unter Anwendung eines direkt-demokratischen Verfahrens) geschehen ist. $^{34)}$

31. Eine Landsgemeinde hatten AI, AR, GL, NW, OW, UR und SZ, halbdirekte Demokratien waren BL, GR, LU, SG, VS und ZG, während AG, BE, BS, FR, GE, SH, SO, TG, TI, VD und ZH repräsentative Demokratien hatten. Siehe G. ANDREY (1983, S. 267).

32. Dementsprechend entwickelten sich in der Schweiz auch das Verwaltungsrecht und die Verwaltungsgerichtsbarkeit erst später als in Deutschland. Zur Entwicklung in Deutschland siehe C. KELLER (1998).

33. Daran ändert sich auch dann nichts, wenn man sich auf außermenschliche Autoritäten beruft. Auch dann, wenn alle Betroffenen sich auf eine göttliche Autorität berufen und/oder die Auffassung vertreten, dass bestimmte Rechte den Menschen ,von Natur aus` zukommen, müssen die entsprechenden Rechtsnormen, damit sie gesellschaftlich wirksam werden, durch Menschen gesetzt oder zumindest, wenn sie durch die Tradition überliefert sind, von ihnen akzeptiert und durchgesetzt werden.

34. Siehe hierzu z.B.: Scharfe Kritik am Fürsten: Bericht von Europarats-Delegierten erschüttert Liechtenstein, NZZ am Sonntag vom 14. September 2003, S. 15; Drohende Überwachung des Fürstentums: Liechtensteins Verfassungsstreit vor dem Europarat, Neue Zürcher Zeitung Nr. 250 vom 28. Oktober 2003, S. 17. 
[32] Sieht man einmal von diesem Kompetenzkompetenz-Problem ab, dann stellt sich die Frage, welcher Spielraum dem Verfassungsgericht eingeräumt werden soll. Als ,Ecklösungen` können die institutionellen Lösungen in Deutschland und in der Schweiz betrachtet werden: Während das Verfassungsgericht in Deutschland, wenn es einmal angerufen wurde, einen sehr weiten Entscheidungsspielraum hat, ist es in der Schweiz nur partiell existent. Der weite Spielraum in Deutschland hat zwei (institutionelle) Ursachen: Zum einen dürfen bestimmte Gruppen, auch wenn sie selbst gar nicht betroffen sind, das Gericht anrufen. Insbesondere hat die (parlamentarische) Opposition das Recht dazu. Dies führt seit den Zeiten des Bundeskanzlers KonRAd AdenAuer dazu, dass die im Bundestag unterlegene Opposition häufig versucht, über das Bundesverfassungsgericht eine Entscheidung der Parlamentsmehrheit annullieren zu lassen und ihre eigene Auffassung zumindest partiell durchzusetzen. Zweitens hat das Gericht die Kompetenz der abstrakten Normenkontrolle, d.h. es kann darüber urteilen, ob ein Gesetz verfassungswidrig ist, selbst wenn bisher überhaupt niemand durch dessen Anwendung in seinen/ihren verfassungsmäßigen Rechten beschnitten wurde. Gleichzeitig kann es in der Urteilsbegründung weitgehende Vorgaben für die zu erlassene gesetzliche Regelung machen. Dadurch wird in bestimmten Fragen das Parlament als Gesetzgeber weitgehend ausgehebelt.

[33] Die schweizerische Alternative dazu besteht nicht nur darin, dass die Verfassungsgerichtsbarkeit auf Bundesebene nur rudimentär existiert. Hinzu kommt, dass nur direkt Betroffene eine Klage einreichen können und dass das Bundesgericht, soweit es überhaupt zuständig ist, nur zur konkreten Normenkontrolle befugt ist: Es kann, wenn es angerufen wird, feststellen, dass bzw. ob der Kläger/die Klägerin in seinen/ihren verfassungsmäßigen Rechten beeinträchtigt wurde. Dies kann auch dazu führen, dass bestimmte gesetzliche Regelungen aufgehoben werden müssen. Letzteres geschah z.B., als das Bundesgericht entschied, dass Einbürgerungsentscheidungen an der Urne dem Willkürverbot in der Verfassung widersprechen und damit unzulässig sind, da für solche Entscheidungen keine Begründungen möglich sind, die angefochten werden können. ${ }^{35)}$ In seiner Urteilsbegründung hat das Bundesgericht aber (seiner üblichen Tradition folgend) bewusst darauf verzichtet darzulegen, wie Einbürgerungsentscheidungen korrekt durchzuführen sind. Damit ist z.B. offen geblieben, ob Einbürgerungsentscheidungen in der Gemeindeversammlung zulässig sind. In der konkreten Situation wurde dies vielfach als Nachteil empfunden, weil die betroffenen Kantone und Gemeinden unsicher waren, wie sie sich in Zukunft verhalten sollen. ${ }^{36)}$ Es ermöglichte ihnen aber, unterschiedliche Institutionen zu entwickeln. So ist es z.B. möglich zu verfügen, dass ein Antrag auf Ablehnung einer Einbürgerung in einer Gemeindeversammlung begründet und dass in dieser Versammlung offen abgestimmt werden muss. Ein derartiges ,Experimentieren“ mit unterschiedlichen Modellen wäre nicht möglich, wenn das Bundesgericht z.B. vorgegeben

35. Siehe hierzu: Zwei Machtworte zur Einbürgerung: Diskriminierung verboten - Begründung erforderlich, Neue Zürcher Zeitung Nr. 157 vom 10. Juli 2003, S. 11; sowie: Ende der Urnen-Einbürgerung, St. Galler Tagblatt vom 10. Juli 2003, S. 5.

36. Siehe hierzu: Kein rechtsfreier Raum bei Einbürgerungen: Bundesgericht begründet Urteile gegen Urnenentscheide, Neue Zürcher Zeitung Nr. 170 vom 25. Juli 2003, S. 13. 
hätte, dass es auch unzulässig ist, über Einbürgerungen in einer Gemeindeversammlung abzustimmen.

[34] Man kann sich fragen, inwieweit Einschränkungen individueller Freiheitsrechte durch Volksentscheide tatsächlich ein relevantes Problem darstellen, oder ob es sich hier nur um rein theoretische Überlegungen handelt, die in der Praxis keine Rolle spielen. Hierzu gibt es einige empirische Arbeiten, die untersuchen, inwiefern direkte Volksrechte zur Einschränkung oder Ausweitung individueller Freiheitsrechte geführt haben. ${ }^{37)}$ Die Ergebnisse sind uneinheitlich, und sie hängen stark von der Einschätzung ab, welche Initiativen bzw. Referenden die Volksrechte einschränken. Insofern kann man diese Frage anhand dieser Studien kaum eindeutig beantworten. Andererseits zeugen die oben aufgeführten Beispiele, dass die Frage der Vereinbarkeit von Volksentscheiden und individuellen Freiheitsrechten in der Schweiz in jüngerer Zeit an Bedeutung gewonnen hat. Dies spricht jedoch nicht gegen diese Volksrechte, sondern allenfalls dagegen, wie diese Fragen in der Schweiz behandelt werden. Man könnte (und sollte wohl auch) dieses Problem lösen, indem man eine verfassungsgerichtliche Überprüfung von Initiativen einführt, um zu verhindern, dass mit den individuellen Menschenrechten nicht verträgliche Initiativen zur Abstimmung gelangen. Zudem kann es, wie das deutsche Beispiel in den siebziger Jahren oder die jüngste Entwicklung in den Vereinigten Staaten zeigen, auch in rein repräsentativen Systemen massive Einschränkungen individueller Freiheitsrechte geben. Diese Gefahr ist nicht auf direkte Demokratien beschränkt.

[35] Diese Überlegungen legen es nahe, dass der ,optimale‘ Kompetenzbereich des Verfassungsgerichts zwischen der deutschen und der schweizerischen Regelung liegen dürfte. Während das Verfassungsgericht in Deutschland eher zu viele Befugnisse hat, herrscht in der Schweiz diesbezüglich ein Defizit. Zur vorhandenen Verfassungsgerichtsbarkeit, die die Kantone betrifft, sollte man insbesondere auch Bundesgesetze dieser Gerichtsbarkeit unterstellen. Um eine Entwicklung wie in Deutschland zu verhindern, sollte man jedoch - wie bisher - nur die konkrete Normenkontrolle zulassen.

\subsection{Der Finanzvorbehalt}

These 5: Die zentralen Instrumente der direkten Demokratie im Bereich der öffentlichen Finanzen, insbesondere das Finanzreferendum, unterstützen eine solide Finanzpolitik. Dagegen ist ein Finanzvorbehalt sinnlos. Dennoch kann auch der Einsatz direktdemokratischer Elemente zu einer Krise der öffentlichen Finanzen führen. Diesbezüglich besteht jedoch kein Unterschied zur repräsentativen Demokratie. Sinnvoller als ein Finanzvorbehalt bei bestimmten direkten Volksrechten, insbesondere der Initiative, ist daher die Einführung von Schuldenbremsen, wie sie der Bund und einzelne Kantone eingeführt haben.

37. Siehe hierzu J.C. MAY (1987), T.E. CRONin (1989), B.S. GAmble (1997), T. DonOvan und S. BowLER (1998) für die Vereinigten Staaten oder B.S. FREY und L. GOETTE (1998) für die Schweiz. 
[36] Es gibt die Befürchtung, dass die Bevölkerung Maßnahmen beschließen könnte, ohne die Möglichkeiten ihrer Finanzierung zu berücksichtigen, und dass sich daraus Probleme für die öffentlichen Haushalte ergeben könnten. Dies kann nicht a priori ausgeschlossen werden, und es liefert die Begründung für den Finanzvorbehalt, den die Verfassungen aller deutschen Bundesländer kennen: Haushaltsrelevante Entscheidungen bleiben dem Parlament vorbehalten und dürfen nicht Gegenstand eines Volksentscheids sein. ${ }^{38)}$ Da es nur wenige politische Entscheidungen gibt, die überhaupt keine finanziellen Auswirkungen haben, bedeutet dies eine massive Einschränkung der direkten Volksrechte.

[37] Ist dieser Vorbehalt gerechtfertigt? Ist diese theoretisch existierende Möglichkeit praktisch relevant? Eine erste Antwort darauf gibt ein Vergleich zwischen der Schweiz und Deutschland: Während in der Schweiz, in der es keinen solchen Vorbehalt gibt, bisher kein Kanton eine Haushaltsnotlage erklärt und von den anderen Kantonen und/oder vom Bund zusätzliche Mittel verlangt hat, haben dies in Deutschland sowohl Bremen als auch das Saarland im Jahr 1992 getan, und sie haben mit ihrer Klage beim Bundesverfassungsgericht Erfolg gehabt. Das Land Berlin hat es im Jahr 2006 ebenfalls versucht, ist aber abgewiesen worden. Bremen und das Saarland verlangen inzwischen weitere Hilfen. Anscheinend ergeben sich in deutschen Bundesländern trotz Finanzvorbehalt massive Finanzschwierigkeiten, während vergleichbares in der Schweiz auch ohne Finanzvorbehalt nicht auftritt.

[38] Ein wesentlicher Grund, weshalb sich die Situation in der Schweiz anders darstellt, obwohl einige Kantone, insbesondere Genf und das Waadtland, sehr stark verschuldet sind, ist die Steuerhoheit der Kantone: Wenn entsprechende Probleme auftreten, können die Kantone ihre Steuereinnahmen erhöhen. Da die Bürgerinnen und Bürger wissen, dass sie im Zweifelsfall mit ihren Steuern für die öffentlichen Ausgaben aufkommen müssen, sind sie dabei entsprechend zurückhaltend. ${ }^{39)}$ Die deutschen Bundesländer haben keine derartige Steuerkompetenz. Dementsprechend könnte es für deren Stimmbürger auch weniger Hemmungen geben, Ausgaben zu beschließen.

[39] Diese Argumentation deckt jedoch nur einen Teil ab. Sie reduziert die direkte Demokratie zumindest implizit auf das Instrument der Initiative (bzw. des Volksbegehrens und des Volksentscheids), was aus der deutschen Perspektive zwar verständlich ist, weil dort nur diese Instrumente zur Verfügung stehen, was aber wesentliche Aspekte ausklammert. Das wichtigste direktdemokratische Instrument der Finanzpolitik ist das Finanzreferendum: Ab einem bestimmten Betrag müssen Ausgaben, die nicht durch Gesetze gebunden sind, entweder automatisch oder auf Antrag, d.h. wenn entsprechend viele Unterschriften zusammen kommen, den Stimmbürgern zur Zustimmung vorgelegt werden. Dadurch können die öffentlichen Ausgaben nur verringert, aber nicht erhöht werden; ein Finanzvorbehalt erübrigt sich gegen dieses Instrument. Das zweite wichtige Instrument ist das (fakultative) Gesetzesreferendum: Neue

38. Siehe hierzu z.B. J. KRAFCZYK (2005).

39. So zeigen z.B. L.P. FELD und G. KIRCHGÄSSNER (2007), dass in den Schweizer Kantonen stärker ausgebaute direkte Volksrechte - ceteris paribus - zu signifikant geringeren kantonalen Schulden pro Kopf führen. 
Gesetze oder Gesetzesrevisionen müssen den Stimmbürgern zur Genehmigung vorgelegt werden, falls diese das wünschen. Auch dieses Instrument dämpft eher die Ausgaben, als dass es sie erhöht, wenn nämlich vom Gesetzgeber beschlossene ausgabenwirksame Gesetze vom Volk verworfen werden. Tatsächlich zeigen empirische Untersuchungen, dass eine Vorlage ceteris paribus - umso eher verworfen wird, je kostspieliger sie ist. ${ }^{40)}$ Es ist freilich auch möglich, dass eine Vorlage, die Einsparungen bringen soll, vom Volk verworfen wird. Insofern kann es gelegentlich auch vorkommen, dass ein erfolgreiches Referendum eine solide Finanzpolitik erschwert.

[40] Deswegen Gesetzesreferenden mit finanziellen Auswirkungen dem Finanzvorbehalt zu unterstellen und damit grundsätzlich zu untersagen, ist schon deshalb nicht angebracht, weil dieses Instrument, wie gesagt, in aller Regel dämpfend auf die Staatsausgaben und damit auch auf die öffentlichen Schulden wirkt. Viel sinnvoller ist es, auf den Ebenen des Bundes wie auch der untergeordneten Gliedkörperschaften, der Bundesländer bzw. Kantone wie auch der Gemeinden, ,Schuldenbremsen` einzuführen, wie sie eine Reihe von Kantonen aber auch die Schweizerische Eidgenossenschaft kennen. Wie empirische Ergebnisse zeigen, haben Kantone mit Schuldenbremsen signifikant niedrigere Defizite und Schulden als Kantone, die keine solchen Institutionen kennen. ${ }^{41)}$ Auch für Deutschland wird im Rahmen der Föderalismusreform II derzeit an die Einführungen solcher Institutionen gedacht. ${ }^{42)}$ Es ist somit möglich, Gefährdungen der finanziellen Solidität auch der untergeordneten Gliedkörperschaften zu begegnen, ohne jene direkten Volksrechte, von denen solche Gefährdungen ausgehen könnten, deshalb einschränken zu müssen. Und da, wie der Vergleich zwischen Deutschland und der Schweiz zeigt, diese Solidität in einem System mit weitaus geringeren Volksrechten sehr viel stärker gefährdet erscheint als in der direkten Demokratie der Schweiz, empfiehlt es sich, solche Bremsen ganz unabhängig davon einzubauen, wie stark diese Volksrechte ausgebaut sind.

\subsection{Direkte Demokratie und politische Reformen}

These 6: In der direkten Demokratie (der Schweiz) besteht eine „Status-Quo-Verzerrung“ durch das Referendum, indem leichter gegen eine Vorlage mobilisiert werden kann als für eine Vorlage. Dies kann auch zu einer Verzögerung von Reformen führen. Bei der Beurteilung dieses Effekts sind jedoch zwei Punkte zu berücksichtigen:

a) Insbesondere bei Verfassungsfragen macht ein starkes Gewicht für den Status Quo Sinn: Praktisch alle Staaten sehen hierfür relativ hohe Quoren vor. Vergli-

40. Siehe hierzu G. KIRCHGÄSSNER und T. SCHULZ (2005).

41. Siehe hierzu, aber auch zu den unterschiedlichen Regelungen auf kantonaler bzw. auf eidgenössischer Ebene L.P. FELD und G. KIRCHGÄSSNER (2007).

42. Siehe hierzu z.B. SACHVERSTÄNDIGENRAT ZUR BEGUTACHTUNG DER GESAMTWIRTSCHAFTLICHEN ENTWICKLUNG (2007). Dabei stehen nicht die kantonalen Regelungen, sondern die Schuldenbremse des Bundes als Vorbild zur Diskussion. 
chen damit sind die Hürden für Verfassungsänderungen in der Schweiz relativ gering.

b) Betrachtet man die jüngste Vergangenheit, hat die Schweiz kaum einen höheren Reformstau als ihre Nachbarstaaten, die rein parlamentarische Demokratien sind. Insofern dürften die Auswirkungen der Status-Quo-Verzerrung tragbar sein.

[41] Wie gerade ausgeführt wurde, kann das fakultative Gesetzesreferendum nicht nur dazu führen, dass ausgabenträchtige Gesetze nicht erlassen werden, sondern es kann auch notwendige Reformen blockieren. Dieser Vorwurf wird von denjenigen, die die direkten Volksrechte in der Schweiz einschränken wollen, immer wieder erhoben. So schreibt zum Beispiel Borner: „Die entscheidende Wirkung des fakultativen Referendums liegt darin, dass dieser Mechanismus der Regierung und dem Parlament jede größere Veränderung des institutionellen Rahmens stark erschwert. ... Umfassende politische oder wirtschaftliche Veränderungen sind in der Schweiz die Ausnahme.“43) Ganz ähnlich, wenn auch noch viel dramatischer, äußert sich W. WitTMAnN (1998). Er fordert: „Die Schweiz muss aus der direkten Demokratie aussteigen, sich wie andere Länder der parlamentarischen Demokratie zuwenden“ (S. 233), da nach seiner Auffassung „,... die direkte Demokratie im Allgemeinen und das Referendum im Besonderen auf Dauer die schweizerische Wirtschaft ruinieren werden, indem sie marktwirtschaftliche Reformen verhindern. Die direkte Demokratie ist auf dem Wege, die Schweiz zu gefährden und zu zerstören.“ (S. 206f.)

[42] Nun lässt sich kaum bestreiten, dass durch das Referendum wichtige Entscheidungen verzögert wurden. So wurde z.B. am 1. Februar 1959 von der Mehrheit der männlichen Stimmbürger zum letzten Mal das Frauenstimmrecht auf Bundesebene abgelehnt; die Frauen bekamen dieses Recht erst am 7. Februar 1971. Noch bedenklicher sah es auf kantonaler Ebene aus. Während die meisten Kantone, soweit sie das nicht schon davor getan hatten, das kantonale Frauenstimmrecht kurz nach der Abstimmung vom Februar 1971 einführten, weigerten sich die beiden Appenzell weiterhin. In Appenzell Ausserrhoden wurde es erst auf der Landsgemeinde im Jahr 1989 eingeführt, und die Innerrhoder Landsgemeinde lehnte es noch im Jahr 1990 ab. Erst eine im gleichen Jahr ergangene Entscheidung des Bundesgerichts, die auch diesen letzten Kanton zur Einführung des Frauenstimmrechts zwang, stellte sicher, dass Frauen auf allen Ebenen in ihren politischen Rechten den Männern gleichgestellt wurden.

[43] In der politischen Diskussion der jüngeren Zeit war es aber weniger dieser Anachronismus, sondern es waren bestimmte wirtschaftspolitische Entscheidungen, wie z.B. die Ablehnung des Beitritts zum EWR am 6. Dezember 1992 oder die Ablehnung der Reform des Arbeitsgesetzes am 1. Dezember 1996, die eine Rolle spielten. Insbesondere die Ablehnung des EWR-Beitritts hatte negative wirtschaftliche Folgen für die Schweiz, und die Politik der bilateralen Verträge, die seitdem eingeschlagen und in der Abstimmung vom 8. Februar 2009

43. S. BoRNER, Direkte Demokratie - letzter Schweizer Mythos, Neue Zürcher Zeitung Nr. 1 vom 3./4. Januar 1997, S. B9f. 
vom Volk eindrücklich bestätigt wurde, konnte dies zwar weitgehend, aber nicht vollständig kompensieren. Daher ist die Frage berechtigt, ob die direkte Demokratie der Schweiz nicht ein Hindernis für die weitere wirtschaftliche Entwicklung darstellen könnte.

[44] Es ist freilich hoch problematisch, solche Fragen anhand des Ausgangs einer einzelnen Abstimmung oder auch einiger weniger Abstimmungen entscheiden zu wollen. Auch in rein repräsentativen Systemen gibt es einzelne Entscheidungen, die negative wirtschaftliche Konsequenzen nach sich ziehen. Die relevante Frage ist, ob die direkte Demokratie im Vergleich mit einem realen rein repräsentativen System zu im Durchschnitt ,besseren` Entscheidungen führt, wobei der Maßstab für ,besser` und ,schlechter` erst noch festgelegt werden müsste. Was Reformen anbetrifft, kann sich die Schweiz zumindest in jüngerer Zeit durchaus mit ihren größeren Nachbarstaaten messen: Reformstaus gibt es in Deutschland, Frankreich und Italien mindestens so sehr wie in der Schweiz. ${ }^{44)}$ So hat die Schweiz z.B. in den letzten Jahren eine Reform ihres Föderalismus vollzogen, die sehr viel weitgehender ist, als alle Reformen, die in Deutschland überhaupt ernsthaft in Erwägung gezogen wurden. ${ }^{45)}$ Dabei sind die deutschen Probleme seit langem bekannt: F.W. SCHARPF hat bereits im Jahr 1985 auf die ,Verflechtungsfalle' hingewiesen, der die deutsche Politik ausgesetzt ist. Das Problem des Reformstaus kann daher nicht auf die Frage rein-repräsentative versus halb-direkte Demokratie reduziert werden.

[45] Andererseits gibt es durch das Referendum eine ,Status-Quo-Verzerrung`, d.h. der Status Quo hat bei einer Abstimmung unter sonst gleichen Bedingungen einen Vorteil. G. KIRCHGÄSSNER und T. SCHULZ (2005) haben gezeigt, dass in einem Abstimmungskampf ein Franken, der zur Werbung gegen eine Vorlage eingesetzt wird, fast die doppelte Wirkung hat wie ein Franken, der zur Unterstützung eingesetzt wird. Dies entspricht auch den Ergebnissen von E.R. GERBER (1999) für die Vereinigten Staaten. Sie zeigt, dass es für Interessengruppen leichter ist, gegen als für eine Vorlage zu mobilisieren. ${ }^{46)}$

[46] Dieser Verzerrung, die durch das Referendum bewirkt wird, steht jedoch die beschleunigende Wirkung der Initiative gegenüber. Sie ermöglicht, Probleme auf die politische Tagesordnung zu setzen, die von den Parteien (bisher) negiert werden. So konnte die schweizerische Bevölkerung z.B. bereits im Jahr 1979 über ein Moratorium für die Kernenergie abstimmen, als in Deutschland noch alle im Bundestag vertretenen Parteien nahezu geschlossen für diese Energieform eintraten. In Deutschland benötigte man die Gründung einer neuen Partei, die sich speziell dafür einsetzte, in der Schweiz ,nur` eine Initiative, um dieses Problem in die ernsthafte politische Diskussion zu bringen.

44. Zur Notwendigkeit von Reformen in Deutschland siehe z.B. die Beiträge in K.F. ZiMMERMANN (2006).

45. Zu den Problemen des deutschen Föderalismus siehe z.B. G. KIRCHGÄSSNER (2007); zur Reform in der Schweiz z.B. C. SCHALTEGGER und R.L. FreY (2003) oder G. KIRCHGÄSSNER (2006).

46. Ähnliche Ergebnisse für die Schweiz, die sich jedoch auf einzelne Abstimmungen beziehen, finden sich bei H.P. HeRTiG (1982), E. GRUNER und H.P. HERTIG (1983) sowie bei C. LONGCHAMP (1991). 
[47] Insofern kennt die direkte Demokratie in der Schweiz beides, eine Bremse und ein Gaspedal. Nun kann man argumentieren, dass das Problem darin liegt, dass die Bremse stärker bzw. zu stark ist. Dies mag zumindest für wirtschaftspolitische Reformen gelten. Schließlich wird kaum eine solche Reform durch eine Initiative gefordert und durchgesetzt, aber mehrere Reformen sind in der jüngeren Vergangenheit am Referendum gescheitert. Aber selbst wenn man dies so sieht, stellt sich die Frage, ob es nicht trotzdem sinnvoll ist, dem Status Quo eine bestimmte Priorität einzuräumen.

[48] Für Verfassungsfragen (und damit für das obligatorische Referendum bei Verfassungsänderungen in der Schweiz) dürfte dies unstrittig sein. Schließlich verlangen alle Länder dafür höhere Quoren als die einfache Mehrheit; in Deutschland müssen sowohl Bundestag als auch Bundesrat mit jeweils zwei Dritteln zustimmen. Dies ist eine sehr viel höhere Hürde als in der Schweiz, die bei einer Verfassungsinitiative nur die Mehrheit der Abstimmenden (Volksmehr) sowie Mehrheiten in der Mehrheit der Kantone (Ständemehr) verlangt. Nicht zufällig wird die Schweizerische Bundesverfassung sehr viel häufiger geändert als die Verfassungen Deutschlands oder gar der Vereinigten Staaten. Insofern könnte man sogar argumentieren, dass das retardierende Element bei Verfassungsfragen in der Schweiz zu gering ist.

[49] Etwas anders sieht es beim fakultativen Gesetzesreferendum aus. Hier kann man argumentieren, dass der Status Quo gegenüber neuen Vorschlägen nicht bevorzugt sein sollte. Auch dieses Argument ist jedoch nicht unproblematisch. Zum einen wird gerade aus der Sicht der Wirtschaft häufig gefordert, dass die Politik stetig und berechenbar sein sollte. Die StatusQuo-Verzerrung verstetigt die Politik. Offensichtlich gibt es ein Spannungsverhältnis zwischen Berechenbarkeit und der Politik und ihrer Offenheit für neue Lösungen. Wie dies optimal aufgelöst werden sollte, ist nicht a priori gegeben. Ein gewisser, nicht zu starker StatusQuo-Bias kann hier durchaus von Vorteil sein.

[50] Zweitens aber ist die entscheidende Frage aus demokratietheoretischer Sicht nicht die, ob der Status Quo einen Vorteil hat, sondern welches System Lösungen hervorbringt, die näher an den Präferenzen der Bevölkerung sind. In dieser Hinsicht hat das direkt-demokratische System eindeutige Vorteile. ${ }^{47)}$ Wenn Reformen in diesem System tatsächlich länger dauern sollten als in rein repräsentativen Systemen, dann sind dies aus demokratietheoretischer Sicht Kosten dafür, dass die Präferenzen der Bevölkerung besser berücksichtigt werden. Und es ist völlig offen, ob dies wirtschaftliche Nachteile mit sich bringt. Untersuchungen, die sowohl für die Bundesstaaten der Vereinigten Staaten als auch für die Schweizer Kantone durchgeführt wurden, zeigen das Gegenteil: Stärker ausgebaute direkte Volksrechte führen - ceteris paribus - zu einer besseren wirtschaftlichen Entwicklung. Man mag einwenden, dass diese Untersuchungen sich nur auf die Ebene der Bundesstaaten und Kantone beziehen und dass es offen ist, ob diese Ergebnisse auf die nationale Ebene übertragen werden können. Es gibt gute Argumente dafür, auch wenn es gelegentlich in Zweifel gezogen wird. Auf jeden Fall aber ist die Entwicklung der Schweiz im Vergleich mit ihren Nachbarstaaten auch in jüngerer Zeit (1999). 
nicht so verlaufen, dass man daraus folgern müsste, dass die direkte Demokratie auf Dauer erhebliche wirtschaftliche Nachteile mit sich bringt. ${ }^{48)}$

\section{Abschließende Bemerkungen}

[51] Es ist offensichtlich: Die direkte Demokratie kennt Probleme. Dies gilt für die Art und Weise, wie sie in der Schweiz praktiziert wird, aber auch für alle anderen Varianten: Bestimmte Probleme sind systemimmanent. Aber auch jedes andere politische System kennt Probleme, auch die rein repräsentative Demokratie. Wenn nach Winston CHURChILL die Demokratie die schlechteste aller Staatsformen ist, abgesehen von allen anderen, die von Zeit zu Zeit ausprobiert wurden, dann ist die direkte Demokratie möglicherweise die schlechteste Form der Demokratie, abgesehen von allen anderen. Schließlich speist sich das heute in vielen Ländern festzustellende Bedürfnis nach mehr direkter Mitsprache der Bürgerinnen und Bürger im politischen Prozess häufig aus Mängeln des repräsentativen Systems.

[52] Dies bedeutet jedoch weder, dass jede Ausgestaltung der direkten Demokratie sinnvoll wäre, noch, dass man Volksabstimmungen beliebig ansetzen sollte. Damit direkte Demokratie sinnvoll funktioniert, ist u.a. auf folgendes zu achten:

(i) Die direkte Demokratie muss als ein System begriffen werden, in welchem die Bürgerinnen und Bürger die ihnen zustehenden Rechte von sich aus ergreifen (können). Dies ist in einen Prozess einzubetten, in welchem der Abstimmung ein intensiver gesellschaftlicher Diskussionsprozess vorangehen kann, so dass die Abstimmenden hinreichend informiert sein können. In diesem Prozess sollten bei Initiativen auch ,Gegenvorschläge‘ von Regierung und das Parlament eingebracht werden können. Von Zeit zu Zeit angesetzte Volksabstimmungen, bei denen den Bürgerinnen und Bürgern von oben ,großzügig‘ erlaubt wird, über einzelne Fragen mitzuentscheiden, machen dagegen kaum Sinn. Sie werden häufig für andere Belange instrumentalisiert, und auch der gesellschaftliche Diskurs dreht sich dann möglicherweise um ganz andere Probleme als die zur Entscheidung anstehende Frage.

(ii) Die direkte Demokratie muss institutionell so ausgestaltet werden, dass sie einerseits handhabbar ist und andererseits nicht zu Blockaden führt. So ist auf Beteiligungsquoren zu verzichten; dort, wo man Quoren für sinnvoll hält, sollten dies Zustimmungsquoren sein. Außerdem muss auf Einstimmigkeit verzichtet werden; sie verträgt sich kaum mit der direkten Demokratie. Dies gilt insbesondere für die Europäische Union. Wenn dort in Zukunft Verfassungsfragen den Bürgerinnen und Bürgern zur Abstimmung vorgelegt werden sollen, sollte vom Einstimmigkeitsprinzip auf qualifizierte Mehrheiten der Bevölkerung wie der Staaten übergegangen werden.

48. Dies gilt trotz der Wachstumsschwäche der Schweiz in den neunziger Jahren und in der ersten Hälfte dieses Jahrzehnts. Siehe hierzu G. KIRCHGÄSSNER (2005). Zur Frage, inwieweit die direkte Demokratie Reformen entgegensteht, siehe auch G. KIRCHGÄSSNER (2008). 
(iii) Konflikte mit individuellen Freiheitsrechten bzw. mit internationalem Recht, die bei Initiativen auftreten können, sollten nach Möglichkeit vermieden bzw. dann, wenn sie auftreten, über eine sinnvoll ausgestaltete Verfassungsgerichtsbarkeit geregelt werden. Es ist für die Beteiligten frustrierend und der politischen Bereitschaft, mitzuwirken, abträglich, wenn Initiantinnen oder Initianten sich für eine Sache einsetzen, die Zustimmung der Stimmbürger gewinnen und dann erfahren müssen, das ihr Anliegen wegen Konflikten mit internationalem Recht nicht umgesetzt werden kann. Dies kann man vermeiden, indem man verfassungsrechtliche Prüfungen frühzeitig vorsieht und damit die Möglichkeit bietet, Initiativen so zu formulieren, dass sie nicht in Konflikt mit internationalem Recht geraten.

Wird dies alles berücksichtigt, dann dürften die mit den direkten Volksrechten verbundenen Probleme ihre Vorteile kaum aufwiegen. Man muss dann auch keine Angst haben, dass Reformen an ihr scheitern müssen, und ein Finanzvorbehalt erübrigt sich, wenn in den einzelnen Gliedkörperschaften sinnvolle Regeln zur Verhinderung übermäßiger Verschuldung eingeführt werden.

\section{Zusammenfassung}

Es gibt im Zusammenhang mit Volksentscheiden verschiedene Probleme, die zum Teil nicht gesehen werden. Auch wird teilweise extrem naiv (und/oder ideologisch) argumentiert. In dieser Arbeit werden einige dieser Probleme diskutiert. Zum einen geht es um die Funktionsweise der direkten Demokratie. Dabei geht es zunächst einmal darum, deutlich zu machen, dass eine direkte Demokratie nur dann ein sinnvolles politisches System darstellt, wenn sie als dauerhafter Prozess ausgestaltet ist und man nicht nur gelegentlich einzelne Fragen den Bürgerinnen und Bürgern zur Abstimmung vorlegt. Danach wird die vor allem für den Bereich der Europäischen Union bedeutsame Frage diskutiert, wie sich die direkte Demokratie zur Einstimmigkeitsregel verhält, bevor die Rolle von Quoren behandelt wird. Danach geht es um tatsächliche oder vermeintliche Konfliktfelder, die sich durch die direkten Volksrechte ergeben. So sind bei Initiativen Konflikte mit dem Völkerrecht möglich; sie treten in der Schweiz gelegentlich auf. Zudem könnten Initiativen zu Finanzproblemen führen, während Referenden Reformen erschweren können. All dies rechtfertigt jedoch nicht, direkte Volksrechte generell abzulehnen. Daher schließt die Arbeit mit einer Auflistung jener wichtigen Punkte, die sich aus unserer Diskussion ergeben und zu beachten sind, damit ein direkt-demokratisches System sinnvoll funktionieren kann. 


\section{Literaturverzeichnis}

Andrey, Georges (1983), Auf der Suche nach dem neuen Staat (1798 - 1848), in: Comité POUR UNE NouVELLE HISTOIRE DE LA SUISSE (ed.), Geschichte der Schweiz - und der Schweizer, Helbing und Lichtenhahn. Basel 1983, S. $177-287$.

Borner, Silvio (2005), Blockierte Schweiz: Wie weiter?, in: L. STEINMANN und H. RENTSCH (2005), S. 201 220.

BöS, Dieter (1989), Arguments on Privatization, in: G. Fels und G.M.v. FürstenBerg (eds.), A Supply-Side Agenda for Germany, Springer, Berlin et al. 1989, S. 217 - 245.

BRUnEtTI, Aymo (1997), Der 'Status-Quo-Bias' und die bremsende Wirkung des fakultativen Referendums, in S. BORNER und H. RENTSCH (eds.), Wieviel direkte Demokratie verträgt die Schweiz, Rüegger, Chur/Zürich 1997. S. $167-181$.

Buchanan, James M., und Yong J. Yoon (2000), Journal of Law and Economics 43 (2000), S. 1 - 13.

Cronin, Thomas E (1989)., Direct Democracy: The Politics of Initiative, Referendum, and Recall, Harvard University Press, Cambridge 1989.

Crow, Robert Thomas (2002), What Works and What Does Not in Restructuring Electricity Markets, Business Economics 37 (2002), S. 41 - 56.

D’Atena, Antonio (1999), Das demokratische Prinzip im System der Verfassungsprinzipien, Jahrbuch des öffentlichen Rechts der Gegenwart, NF 47 (1999), S. 1 - 14.

Donovan, TodD, und SHAwn Bowler (1997), Direct Democracy and Minority Rights: Opinions on Anti-Gay and Lesbian Ballot Initiatives, in: S.L. WITT und S. MCCORKLE (eds.), Anti-Gay Rights: Assessing Voter Initiatives, Praeger, Westport/London 1997, S. 107 - 125.

FEHR, HANS (1962), Deutsche Rechtsgeschichte, Walter de Gruyter, Berlin, 6. Auflage 1962.

FELD, LARS P., und GEBHARD KIRCHGÄSSNER (2007), Zur Effektivität von Schuldenbremsen: Die Erfahrung der Schweiz, Das öffentliche Haushaltswesen in Österreich 48 (2007), S. 267 - 296.

Frey, Bruno S., und Lorenz Goette (1998), Does the Popular Vote Destroy Civil Rights?, American Journal of Political Science 42 (1998), S. 1343 - 1348.

Gamble, Barbara S. (1997), Putting Civil Rights to a Popular Vote, American Journal of Political Science 41 (1997), S. $245-269$.

Gerber, ElisABeth, R. (1996), Legislative Response to the Threat of Initiatives, American Journal of Political Science 40 (1996), S. $99-128$.

Gerber, Elisabeth, R. (1999), The Populist Paradox: Interest Group Influence and the Promise of Direct Legislation, Princeton University Press, Princeton 1999.

Gruner, Erich, und Hans Peter Hertig (1983), Der Stimmbürger und die neue Politik, Haupt, Bern/Stuttgart 1983.

HAYEK, FRIEDRICH August VON (1967), Grundsätze einer liberalen Gesellschaftsordnung, ORDO 18 (1967), S. $11-33$

HÉretier, Adrienne (1999), Policy-Making and Diversity in Europe, Cambridge University Press, Cambridge (UK) et al. 1999.

Hertig, HANS Peter (1982), Sind Abstimmungen käuflich?, Schweizerisches Jahrbuch für Politische Wissenschaft 22 (1982), S. $35-58$.

KelleR, CHRISTIAN, Verwaltungsgerichtsbarkeit, in: A. ERLER und E. KAUfMANN (eds.), Handwörterbuch der deutschen Rechtsgeschichte, Band 5, Erich Schmidt, Berlin 1998, S. 879 - 883.

KIRCHGÄSSNER, GEBHARD (2005), Sind direkte Demokratie und Föderalismus schuld an der Wachstumsschwäche der Schweiz?, in: L. STEINMANN und H. RENTSCH (2005), S. 175 - 199.

KIRCHGÄSSNER, GEBHARD (2006), Jüngere Entwicklungen der Finanzsysteme föderaler Staaten: Der ,Neue Finanzausgleich“ in der Schweiz, in: H. BAUER, H. HANDLER und M. SchrATZENSTALLER (eds.), Finanzmanagement im föderalen Staat: Internationale und nationale Reformansätze, Neuer Wissenschaftlicher Verlag, Wien 2006, S. 51 - 69. 
KIRCHGÄSSNER, GEBHARD (2007), Zur Reform der Finanzbeziehungen zwischen Bund und Ländern: Was kann Deutschland von der Schweiz lernen?, in: B. NEUMÄrKER und C. SchnABEL (eds.), Ordnungspolitik für den öffentlichen Sektor, Metropolis, Marburg 2007, S. 13 - 40.

Kirchgässner, GeBhard (2008), Direct Democracy: Obstacle to Reform?, Constitutional Political Economy 19 (2008), S. $81-93$.

KIRCHGÄSSNER, GEBHARD, und TOBIAS SCHUlz (2005), Erwartete Knappheit oder Mobilisierung: Was führt zu hoher Abstimmungsbeteiligung? Empirische Ergebnisse für die Schweiz, 1981 - 1999, in: J. FALTER, O.W. GABRIEL und B. Wessels (eds.), Wahlen und Wähler: Analysen aus Anlass der Bundestagswahl 2002, Verlag für Sozialwissenschaften, Wiesbaden 2005, S. 515 - 550.

Kirchgässner, GeBhaRd, LARS P. Feld und MARCEL R. SAVIOZ (1999), Die direkte Demokratie: Modern, erfolgreich, entwicklungs- und exportfähig, Helbing und Lichtenhahn/Vahlen, Basel/München 1999.

KRAFCZYK, JÜRGEN (2005), Der parlamentarische Finanzvorbehalt bei der Volksgesetzgebung, Duncker und Humblot, Berlin 2005.

LONGCHAMP, ClAude (1991), Herausgeforderte demokratische Öffentlichkeit: Zu den Möglichkeiten und Grenzen des politischen Marketings bei Abstimmungen und Wahlen in der Schweiz, Schweizerisches Jahrbuch für Politische Wissenschaft 31 (1991), S. 303 - 326.

MATSUSAKA, John G. (1995), Fiscal Effects of the Voter Initiative: Evidence from the Last 30 Years, Journal of Political Economy 103 (1995), S. 587 - 623.

MAY, JANICE C. (1987), Constitutional Amendment and Revision Revisited, Publius 17 (1987), S. 153 - 170.

Mueller, Dennis C. (2003), Public Choice III, Cambridge University Press, Cambrigde (U.K.) 2003.

NiPPERDEY, ThOMAs (1983), Deutsche Geschichte 1800 - 1866: Bürgerwelt und starker Staat, C.H. Beck, München 1983.

Pommerehne, Werner W. (1978), Institutional Approaches to Public Expenditure: Empirical Evidence from Swiss Municipalities, Journal of Public Economics 9 (1978), S. 255 - 280.

Prisching, MANFRed (1988), Privatisierung als symbolische Politik, Wirtschaftspolitische Blätter 35 (1988), S. $408-416$.

SACHVERSTÄNDIGENRAT ZUR BEGUTACHTUNG DER GESAMTWIRTSCHAFTLICHEN ENTWICKLUNG (2007), Staatsverschuldung wirksam begrenzen: Expertise im Auftrag des Bundesministers für Wirtschaft und Technologie, Wiesbaden, März 2007.

SCHAFFNER, MARTIN (1998), ,Direkte‘ oder ,indirekte‘ Demokratie: Konflikte und Auseinandersetzungen, 1830 - 1848, in: A. ERnST, A. TANNER und M. WEISHAUPT (eds.), Revolution und Innovation: Die konfliktreiche Entstehung des schweizerischen Bundesstaates von 1848, Chronos, Zürich 1998, S. 271 - 277.

Schaltegger, Christoph, und RenÉ L. Frey (2003), Finanzausgleich und Föderalismus: Zur Neugestaltung der föderativen Finanzbeziehungen am Beispiel der Schweiz, Perspektiven der Wirtschaftspolitik 4 (2003), S. $239-258$.

SCHARPF, FRITZ W. (1985), Die Politikverflechtungsfalle: Europäische Integration und deutscher Föderalismus im Vergleich, Politische Vierteljahresschrift 26 (1985), S. 323 - 356.

SchmacK-ReschKe, Thomas (1997), Bürgerbeteiligung und Plebiszite in der Debatte der Gemeinsamen Verfassungskommission, in: N. KONEGEN und P. NITSCHKE (eds.), Revision des Grundgesetzes? Ergebnisse der gemeinsamen Verfassungskommission (GVK) des Deutschen Bundestages und des Bundesrates, Leske und Budrich, Opladen 1997, S. 77 - 105.

Schnabel, Franz (1964), Deutsche Geschichte im neunzehnten Jahrhundert, Band 3: Monarchie und Volkssouveränität, Herder, Freiburg et al. 1964.

SteinMAnN, LuKAS, und HANS RENTSCH (eds.) (2005), Diagnose: Wachstumsschwäche, Die Debatte über die fehlende Dynamik der Schweizerischen Volkswirtschaft, Verlag Neue Zürcher Zeitung, Zürich 2005.

Stigler, GeORge J. (1979), Why Have the Socialists Been Winning?, ORDO 39 (1979), S. 61 - 68.

StOlleis, Michael (1990), Rechtsstaat, in: A. ERLER und E. KAUFMANN (eds.), Handwörterbuch der deutschen Rechtsgeschichte, Band 4, Erich Schmidt, Berlin 1990, S. 367 - 375. 
VAubeL, Roland (2000), Internationaler Politischer Wettbewerb: Eine europäische Wettbewerbsaufsicht für Regierungen und die empirische Evidenz, Jahrbuch für Neue Politische Ökonomie 19 (2000), S. 280 - 335.

VAuBEL, RolAND (2001), Europa-Chauvinismus: Der Hochmut der Institutionen, Herbig, München 2001.

VAUBEL, RolAnd (2006), Die Konstitutionalisierung der Europäischen Union aus verfassungsökonomischer und politisch-ökonomischer Sucht, in: E. BUSEK und W. HuMMER (eds.), Die Konstitutionalisierung der Verbandsgewalt in der (neuen) Europäischen Union, Böhlau, Wien et al. 2006, S. 97 - 106.

WiCKSELL, KNUT (1896), Finanztheoretische Untersuchungen, Gustav Fischer, Jena 1896.

Wittmann, Walter (1998), Die Schweiz: Ende eines Mythos, Wirtschaftsverlag Langen Müller/Herbig, München 1998.

Zimmermann, Klaus F. (2006) (ed.), Deutschland - was nun?, Reformen für Wirtschaft und Gesellschaft, dtv, München 2006. 